\title{
Residual donors and compensation in metalorganic chemical vapor deposition as-grown $n$-GaN
}

\author{
Xiaoliang $\mathrm{Xu},{ }^{a)}$ Hongtu Liu, and Chaoshu Shi \\ Department of Physics, The University of Science and Technology of China, Hefei, Anhui 230026, \\ People's Republic of China \\ Youwen Zhao \\ P. O. Box 912, Institute of Semiconductors, Chinese Academy of Sciences, Beijing, 10083, \\ People's Republic of China \\ S. Fung ${ }^{\text {b) }}$ and C. D. Beling \\ Department of Physics, The University of Hong Kong, Hong Kong, People's Republic of China
}

(Received 20 March 2001; accepted for publication 22 August 2001)

\begin{abstract}
In our recent report, [Xu et al., Appl. Phys. Lett. 76, 152 (2000)], profile distributions of five elements in the GaN/sapphire system have been obtained using secondary ion-mass spectroscopy. The results suggested that a thin degenerate $n^{+}$layer at the interface is the main source of the $n$-type conductivity for the whole film. The further studies in this article show that this $n^{+}$conductivity is not only from the contribution of nitride-site oxygen $\left(\mathrm{O}_{\mathrm{N}}\right)$, but also from the gallium-site silicon $\left(\mathrm{Si}_{\mathrm{Ga}}\right.$ ) donors, with activation energies $2 \mathrm{meV}$ (for $\mathrm{O}_{\mathrm{N}}$ ) and $42 \mathrm{meV}$ (for $\mathrm{Si}_{\mathrm{Ga}}$ ), respectively. On the other hand, Al incorporated on the Ga sublattice reduces the concentration of compensating Ga-vacancy acceptors. The two-donor two-layer conduction, including Hall carrier concentration and mobility, has been modeled by separating the GaN film into a thin interface layer and a main bulk layer of the GaN film. The bulk layer conductivity is to be found mainly from a near-surface thin layer and is temperature dependent. $\mathrm{Si}_{\mathrm{Ga}}$ and $\mathrm{O}_{\mathrm{N}}$ should also be shallow donors and $\mathrm{V}_{\mathrm{Ga}}-\mathrm{O}$ or $\mathrm{V}_{\mathrm{Ga}}-\mathrm{Al}$ should be compensation sites in the bulk layer. The best fits for the Hall mobility and the Hall concentration in the bulk layer were obtained by taking the acceptor concentration $N_{A}=1.8$ $\times 10^{17} \mathrm{~cm}^{-3}$, the second donor concentration $N_{D 2}=1.0 \times 10^{18} \mathrm{~cm}^{-3}$, and the compensation ratio $C=N_{A} / N_{D 1}=0.6$, which is consistent with Rode's theory. Saturation of carriers and the low value of carrier mobility at low temperature can also be well explained. (C) 2001 American Institute of Physics. [DOI: 10.1063/1.1413706]
\end{abstract}

\section{INTRODUCTION}

$\mathrm{GaN}$ is a III-V semiconductor, with great physical hardness, extremely large heterojunction offsets, high thermal conductivity, and high melting temperature. ${ }^{1} \mathrm{Up}$ to the late 1980 's, there was only modest interest in III-V materials for applications to light emitting diodes (LEDs). However, since then the field has been revolutionized by successes in fabricating blue LEDs from GaN/InGaN. ${ }^{2,3}$ Recently, the study of GaN films becomes an international hot point, ${ }^{4}$ and there has been significant progress in the improvement of crystal quality, $p$-type control, and growth technology. ${ }^{5-7}$

As-grown $\mathrm{GaN}$ is usually $n$-type, and $p$-type conductivity is difficult to obtain., ${ }^{5,6}$ For almost 30 years, the $n$-type conductivity has been commonly associated with the nitrogen vacancy. ${ }^{8,9}$ Although this assignment was consistent with tight-binding calculations, ${ }^{10,11}$ these calculations have limited accuracy, and in addition give no information about atomic defect geometry (relaxation, reconstruction, etc.) and defect formation energies. The formation energy of a defect determines whether a defect can actually form and in what concentration. Recent theoretical calculations ${ }^{12-15}$ shown that

${ }^{a)}$ Electronic mail: lism@ustc.edu.cn

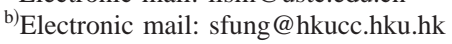

the formation energy of nitrogen vacancy $\left(V_{\mathrm{N}}\right)$ in as-grown $n$-type $\mathrm{GaN}$ film is $\sim 4 \mathrm{eV}$, which is much greater than that of gallium vacancy $\left(V_{\mathrm{Ga}}\right)(\sim 0.7 \mathrm{eV})$. Therefore, the authors proposed that $V_{\mathrm{N}}$ should not exist in $n$-type $\mathrm{GaN}$, but the most probable source of donors is $\mathrm{V}_{\mathrm{Ga}}-\mathrm{O}$ complex or gallium site silicon $\left(\mathrm{Si}_{\mathrm{Ga}}\right.$, with formation energy $\left.\sim 1.0 \mathrm{eV}\right)$. The fact the oxygen as a shallow donor in $n$-type $\mathrm{GaN}$ has been experimentally identified. ${ }^{16-20}$ Oxygen is to be referred from the reaction gas $\mathrm{NH}_{3}$ and the growth circumstance ${ }^{16}$ as a shallow donor, the activation energy ranging from 4-10 $\mathrm{meV}$ is extracted as a function of doped $\mathrm{O}$ concentration. ${ }^{16}$ The study of unintentionally doped Si has not been seen by the authors in published literature but the study of Si-doped $n^{+}$-GaN films can be found in reports of photoluminescence $(\mathrm{PL})^{21}$ and pressure Raman measurements. ${ }^{22}$ The experiments have shown that the intentionally doped silicon can form a shallow donor with different activation energies of $25-35 \mathrm{meV}^{23-25}$

Temperature dependent Hall measurement is a useful method to search the concentration, the activation energy, and the mobility of carriers. Details of the carriers (such as the kind and the type) can also be obtained by combining with other experimental methods. Although studies of the temperature dependent electron transport mechanism in as- 
grown $\mathrm{GaN}$ have been conducted for many years, ${ }^{10,26-29}$ it has been an ambiguity to explain where the residual donor(s) and the compensation come from. There still exist the following problems in the explanations: (1) As is often the case, in fitting the Hall data over a temperature range, equally good fits can be obtained with a wide range of assumptions about compensation ratio $C=N_{A} / N_{D}$. It can be an ambiguity in choosing the $C$ value. The fitted values of $N_{A}$ were often too small. ${ }^{10,26-28}$ (2) As for the compensation ratio $C$, the variation of the estimated or fitted values of $\Delta E_{D}$ has been considerable. The obtained $\Delta E_{D}$ is really an average effect of two kinds of donors as opposed to usually using one donor model in the fitting of the data. ${ }^{10,26-29}$ (3) It has recently been suggested that a thin degenerate layer found at the GaN sapphire interface results from a high concentration of stacking faults. ${ }^{30}$ Because of the large lattice mismatch (14\%) between $\mathrm{GaN}$ and sapphire, a thin, highly dislocated region is generated at the layer/substrate interface to relieve the strain. However, little is known at present about the electrical and optical properties of the interface. The carrier concentration and mobility in this thin layer has a great effect on the main part of the film especially at a low temperature. ${ }^{30,31}$

In this article, the Hall data to be fitted by using a twodonor two-layer model: the carrier conduction, including Hall carrier concentration and mobility, will be modeled by separating the GaN film into the interface thin layer and a main layer of the GaN film. This is based on the correct choosing of the parameters such as the activation energies of the first and the second donor bands, the concentration of the acceptor $N_{A}$, the compensation ratio $C$, etc. The saturation of carriers and the low value of carrier mobility at a low temperature can also be well explained. The further information of the compensation and the residual donors are also obtained by secondary ion-mass spectroscopy (SIMS) study on the GaN film/sapphire. The date suggests not only the degenerate thin layer directly by the SIMS observation, but also the donors coming from nitrogen-site oxygen $\left(\mathrm{O}_{\mathrm{N}}\right)$ and $\mathrm{Si}_{\mathrm{Ga}}$ are most probable shallow donors in as-grown $n-\mathrm{GaN}$.

\section{EXPERIMENT}

\section{A. Sample preparation}

Samples used in the present work were grown by twoflow metalorganic chemical vapor deposition (MOCVD) method, which has been described in a previous work. ${ }^{32}$ In particular, a $300 \AA$ GaN buffer layer was grown between the $\mathrm{GaN}$ film and the sapphire substrate of (0001) orientation. Prior to growing the buffer layer, the substrate was hydrogenated at $1050{ }^{\circ} \mathrm{C}$ (some others used a nitridation method to treatment the substrate, but there is no obvious different in effect between these two methods). The thickness of the GaN films was $2.0 \mu \mathrm{m}$. The electron concentrations of the undoped $n$-GaN films were around $8 \times 10^{17} \mathrm{~cm}^{-3}$, the electron mobility around $200 \mathrm{~cm}^{2}(\mathrm{~V} \mathrm{~s})^{-1}$, and the resistivity around $5 \times 10^{-2} \Omega \mathrm{cm}$, at room temperature.

\section{RESULTS AND DISCUSSION}

The electrical transportation in the GaN samples were characterized by temperature dependent Hall ranging from
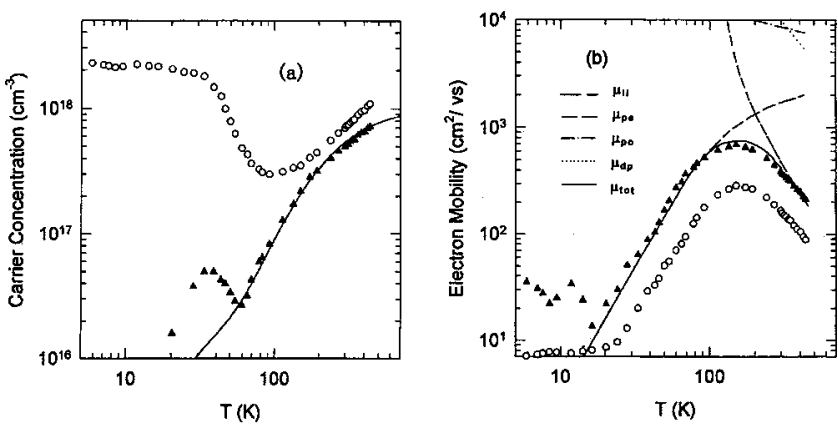

FIG. 1. (a) Temperature dependence of the uncorrected Hall carrier concentration (open circles), and the corrected data (triangles) with the interfacial conduction subtracted. The line shows the model fit to the data. (b) Temperature dependence of the uncorrected electron mobility (open circles), and the corrected values (triangles) after the interfacial conduction effect has been subtracted. The line shows the model fit to the data.

5-430 K. We measured three samples, the data from one sample is shown in Fig. 1. The open circles in Fig. 1 represents the temperature effective carrier concentration [Fig. 1(a)] and mobility [Fig. 1(b)]. In order to obtain a more detailed picture of the defects or impurities present in the $n$-GaN film, we investigated a depth profile of six elements ( $\mathrm{Ga}, \mathrm{N}, \mathrm{O}, \mathrm{Al}, \mathrm{Si}$, and $\mathrm{C}$ ) using SIMS. As shown in Fig. 2, the intensities of the six elements are only relative because of their different SIMS yields. However, an absolute concentration distribution of $\mathrm{Ga}, \mathrm{N}, \mathrm{Al}$, or $\mathrm{O}$ can be obtained due to following approximations: (i) The concentrations of Ga vacancies and $\mathrm{N}$ vacancies $\left(V_{\mathrm{Ga}}\right.$ and $\left.V_{\mathrm{N}}\right)$ in the $\mathrm{GaN}$ film, which are at some distance from the interface region $(\sim 0.2$ $\mu \mathrm{m}$, from the SIMS spectra), are not high (i.e., they are less than $10^{22} \mathrm{~cm}^{-3}$ ). Based on this reasonable assumption, the concentration of $\mathrm{Ga}$ (or $\mathrm{N}$ ) in the film is $4.5 \times 10^{22} \mathrm{~cm}^{-3}$. (ii)

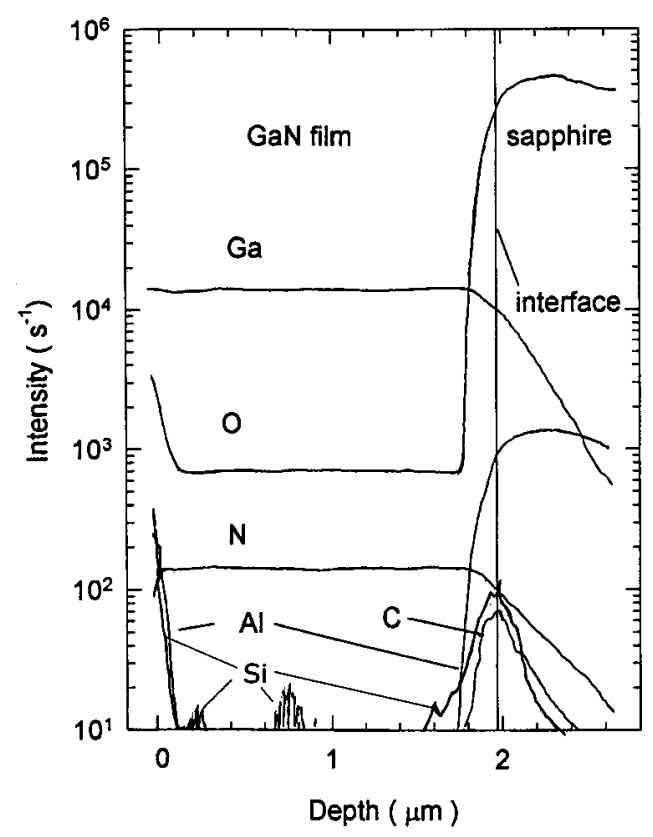

FIG. 2. SIMS depth profile of an $n$-GaN film on a sapphire substrate. The intensities of the six elements are only relative because of their different yields in SIMS. The high intensities of $\mathrm{O}, \mathrm{Al}$, and $\mathrm{Si}$ are due to atomic diffusion and a segregation effect. 
The diffusing source of $\mathrm{Al}$ and $\mathrm{O}$ is from the sapphire substrate. This is due to the fact that the diffusion is associated with the film growth process. ${ }^{32}$ Absolute concentrations of $\mathrm{Al}$ and $\mathrm{O}$ in the film may, therefore, are obtained by referencing to the known values in the sapphire, namely, $4.6 \times 10^{22} \mathrm{~cm}$ and $6.9 \times 10^{22} \mathrm{~cm}^{-3}$, respectively. Based on these assumptions, the calculated average concentrations of $V_{\mathrm{Ga}}, V_{\mathrm{N}}, \mathrm{O}$, and $\mathrm{Al}$ in the interface region are $5 \times 10^{21} \mathrm{~cm}^{-3}, 5$ $\times 10^{22} \mathrm{~cm}^{-3}, \quad 2.4 \times 10^{22} \mathrm{~cm}^{-3}$, and $3.5 \times 10^{22} \mathrm{~cm}^{-3}$, respectively. ${ }^{31}$ These concentrations lead to an expectation that most Ga vacancy sites are replaced by the group-III element $\mathrm{Al}$ at the interface. This follows not only from the copious amount of $\mathrm{Al}$ present at the interface, but also from the fact that $\mathrm{Al}$ has a similar chemistry to Ga. Since the Ga vacancy is believed to be a dominant acceptor in $\mathrm{GaN}$, it then follows that the compensation ratio of the interface layer would be greatly reduced. Turning now to donor sites, we find an opposite tendency. If the main source of the donor is from the $V_{\mathrm{N}}$ or from the $\mathrm{N}$-site oxygen $\mathrm{O}_{\mathrm{N}}$, the donor concentration could be extremely high in the interface layer with the donor sites present at the $10^{21}-10^{22} \mathrm{~cm}^{-3}$ level and with the aforementioned reduction in $V_{\mathrm{Ga}}$ acceptor sites, an extremely high $10^{21} \mathrm{~cm}^{-3}$ electron density would be expected. This electron concentration would be degenerate and temperature independent, since the Mott concentration in GaN is about $1 \times 10^{18} \mathrm{~cm}^{-3},{ }^{33}$ and the concentration at which the Fermi level enters the conduction band is about $6 \times 10^{18} \mathrm{~cm}^{-3} \cdot{ }^{34}$ It is necessary to point out that the only reason the very high depletion of $\mathrm{Ga}$ and $\mathrm{N}$ in the interface region should be referred to the atomic diffusion associated with the growth process of the film as we reported in our previous work. ${ }^{31,32}$ However, $V_{\mathrm{N}}$ and $\mathrm{O}_{\mathrm{N}}$ are difficult to be formed in other regions of the film far from the interface, because the effect of diffusion is greatly reduced, and because of their big formation energies. ${ }^{12-14} \mathrm{As}$ for $\mathrm{Si}$ and $\mathrm{C}$, although the real concentrations are not confirmed, however, we could still propose that $\mathrm{Si}$ and $\mathrm{C}$ exist in high concentrations in the interface layer and the near surface region $(\sim 0.05 \mu \mathrm{m})$, because $\mathrm{Si}$ and $\mathrm{C}$ have similar atomic numbers to $\mathrm{Al}$ and $\mathrm{N}$. Therefore, $\mathrm{Si}_{\mathrm{Ga}}$ should be the main donor source and $\mathrm{V}_{\mathrm{Ga}}-\mathrm{O}$ or $\mathrm{V}_{\mathrm{Ga}}-\mathrm{Al}$ is a probable compensation style in the $n$-GaN film except the interface layer. We find a high oxygen concentration $\left(>10^{19} \mathrm{~cm}^{-3}\right)$ and some $\mathrm{N}$ depletion $\left(\sim 10^{20} \mathrm{~cm}^{-3}\right)$ in the near surface, this may form the first shallow donor $\mathrm{O}_{\mathrm{N}}$. On the other hand, $\mathrm{C}_{\mathrm{Ga}}$ should not be accepted as a donor because its formation energy is as high as $\sim 6.0 \mathrm{eV}^{13}$ As a result of atomic diffusion and segregation, ${ }^{31,32} \mathrm{Al}, \mathrm{O}$, and $\mathrm{Si}$ are also found to be in the interface layer and the surface layer with high concentrations. $\mathrm{Al}$ and $\mathrm{O}$ are from the substrate, ${ }^{31} \mathrm{Si}$ is probably from a quartz glass tube or a growth material, trimethylgallium. The reason why the $\mathrm{N}$ depletion occurs in the near surface is unclear. The phenomenon was neglected in our previous work and is identified in this report. Such depletion in the surface layer was also found in other SIMS data (e.g., see Ref. 35).

The aforementioned arguments explain why, on the basis of atomic interdiffusion during growth, a thin degenerate $n^{+}$ interface layer was formed between the sapphire and the
GaN film. The SIMS data suggest the thickness of this layer to be about $0.2 \mu \mathrm{m}$. The conduction of the other layer of the film $(\sim 1.8 \mu \mathrm{m}$, bulk film) seems to be mainly from another near surface region $(\sim 0.05 \mu \mathrm{m})$ - we can still take this thin region into the whole bulk-film layer, because the conductivity in this region is no degenerate and is temperature dependent. Thus, it is with this thin degenerate interface layer in mind that our focus is now turned to modeling its effect on Hall measurement data.

The temperature-independent mobility $\mu_{2}$ and concentration $n_{2}$ (from the interface layer properties) allow an easy separation of the bulk-film Hall properties, $\mu_{H 1}$, and $n_{H 1}$, from the measured two-layer properties, $\mu_{H}$ and $n_{H} \cdot{ }^{36}$ Using this two-layer model, our Hall data gives an interfacial carrier density $n_{2}=2.2 \times 10^{19} \mathrm{~cm}^{-3}$, which is lower than that obtained from the SIMS results of $\sim 10^{21} \mathrm{~cm}^{-3}$. The most probable explanation is that the $\mathrm{GaN}$ of the interface layer is not of perfect crystal structure. The lattice mismatch and different thermal conductivity between the GaN film and sapphire is so large that a "quasicrystalline" layer formed. ${ }^{30}$ The carrier concentration is lower than that of $V_{\mathrm{N}}$ and $\mathrm{O}_{\mathrm{N}}$ partly because, in such an imperfect layer, only some of the latter are electrically active, and partly because the dislocations present would trap carriers. From Eqs. (1) and (2), ${ }^{30}$ the extracted film concentration $n_{H 1}$, and mobility $\mu_{H 1}$ of a typical sample are plotted in Figs. 1(a) and (b), respectively:

$$
\begin{aligned}
& \mu_{H 1}=\frac{\mu_{H}^{2} n_{H}-\mu_{2}^{2} n_{s h 2} / d}{\mu_{H} n_{H}-\mu_{2} n_{s h 2} / d}, \\
& n_{H 1}=\frac{\left(\mu_{H} n_{H}-\mu_{2} n_{s h 2} / d\right)^{2}}{\mu_{H}^{2} n_{H}-\mu_{2}^{2} n_{s h 2} / d},
\end{aligned}
$$

where $n_{H s h 2}$ is sheet Hall concentration of the interface layer, and $d$ is the whole film thickness.

To fit the carrier concentration data, we use a two-donor model..$^{32}$ Based on PL measurements, ${ }^{37,38}$ the second is a deeper shallow donor with $E_{c}-E_{D 2}=42 \mathrm{meV}$. Figure 3 shows the compensation ratio of our three samples in mobility vs carrier concentration plot from which the corrected room temperature $\mu_{H 1}$ are in good agreement with the compensation ratio $C=0.6$ (for the calculated results of the carrier-concentration-dependent compensation ratio, see Ref. 39 for details). Since three samples have similar Hall and SIMS characterizations, we chose one of them as the analysis target. If we chose $N_{D 1}=3.0 \times 10^{17} \mathrm{~cm}^{-3}$ (which corresponds to the value of the $\mu_{H 1}$ ), one obtains $N_{A}=0.6 N_{D 1}$ $=1.8 \times 10^{17} \mathrm{~cm}^{-3}$. Moreover, the first donor level may be obtained as $E_{C}-E_{1}=5 \times 10^{-15} N_{D 1}^{2 / 3}=2.0 F \mathrm{meV}$ (formula from Refs. 30 and 31). According to Ilegems and Montgomery, ${ }^{9}$ the saturation temperature for complete donor ionization is $T \sim 1000 \mathrm{~K}$. Extension of the measurement data to this temperature suggests a value of $N_{D 2}=1$ $\times 10^{18} \mathrm{~cm}^{-3}$.

Although the concentration of $\mathrm{O}_{\mathrm{N}}$ and $\mathrm{Si}_{\mathrm{Ga}}$ can not be obtained in the interface layer, however, from the SIMS data, a vast of diffused $O$ filled the nitrogen vacancies. Therefore, $\mathrm{O}_{\mathrm{N}}$ donor should be dominant donor in the layer. We can set the concentration of $\mathrm{O}_{\mathrm{N}}$ is $\left[\mathrm{O}_{\mathrm{N}}\right]_{\text {interface }}=2.2 \times 10^{19} \mathrm{~cm}^{-3}$ $=n_{2}$. The effect of $n_{2}$ on the measured Hall carrier concen- 


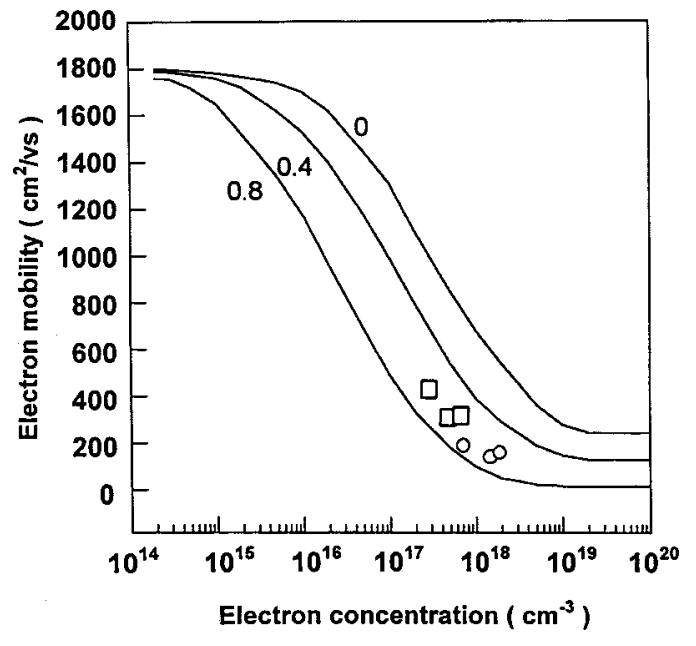

FIG. 3. Comparison of theoretical and experimental values of electron mobility in GaN thin films as a function of free electron concentration. The three thin lines represent calculations by Rode for compensation ratios $C$ $=0,0.4$ and 0.8 . The three open circles correspond to the uncorrected data and three open square to the corrected data of our samples, from which $C$ $\sim 0.60$ were obtained.

tration $n_{H}$ is temperature dependent. When $T>30 \mathrm{~K}$, the $\mathrm{O}_{\mathrm{N}}$ donor is ionized gradually to result in the $n_{H}$ curve reduced while $T$ gets higher-this is in contrast to the saturated constant value of $n_{H}=n_{2} / d$ when $T<30 \mathrm{~K}(k T \sim 2.5 \mathrm{meV}$ for $T=30 \mathrm{~K}$, this is almost equal to the previously calculated 2.0 $\mathrm{meV}$ ). When $T>200 \mathrm{~K}$, the effect of the donors in the bulk layer becomes dominant and the tendency of the $n_{H}$ curve approaches the carrier concentration in the bulk $n_{1}$.

It is found that the corrected mobility data [Fig. 1(b)] are fitted well by solutions of the Boltzmann equations in the relaxation time approximation assuming ionized impurity, deformation potential, piezoelectric, and polar-optical phonon scattering. The good fit is obtained by choosing these values of $E_{C}-E_{1}, E_{C}-E_{2}, N_{A}, N_{D}$, and $n_{c}$ in the calculation. The scattering constants are taken from Ref. 39, and references therein: acoustic potential $E_{1}=9.2 \mathrm{eV}$, piezoelectric constant $h_{p z}=0.5 \mathrm{C} / \mathrm{m}^{2}$, dielectric constant $\epsilon_{\infty}=5.47 \epsilon_{0}$ and $\epsilon_{l f}=10.4 \epsilon_{0}$, effective mass $m^{*}=0.22 m_{0}$, speed of sound $s=6.59 \times 10^{3} \mathrm{~m} \mathrm{~s}^{-1}$, mass density $\rho=6.10$ $\times 10^{3} \mathrm{kgm}^{-3}$, and Debye temperature $T_{D}=1044 \mathrm{~K}$.

In conclusion, the SIMS data suggest a huge material depletion of $\mathrm{Ga}$ and $\mathrm{N}$ and very high concentrations of $\mathrm{O}, \mathrm{Si}$, and $\mathrm{C}$ close to the interface in the $\mathrm{GaN}$ film. It is therefore argued that $\mathrm{N}$ vacancy together with $\mathrm{N}$-site $\mathrm{O}$ could be the first donor, and the Ga-site $\mathrm{Si}$ or $\mathrm{C}$ the second donor, respectively. It is further argued that while the Ga vacancy would tend to compensate for the donors, the compensation ratio could be greatly reduced with $\mathrm{Al}$ diffusing from the sapphire and incorporating at the Ga sites, in the degenerate thin layer. The two-donor two-layer conduction, including Hall carrier concentration and mobility, has been modeled by separating the GaN film into the thin interface layer and a bulk layer of the GaN film. Si, O, and $\mathrm{Al}$ were also found to be as high concentrations and a huge $\mathrm{N}$ depletion found to be in the near surface region $(\sim 0.05 \mu \mathrm{m})$, therefore, $\mathrm{Si}_{\mathrm{Ga}}$ and $\mathrm{O}_{\mathrm{N}}$ should be the shallow donors and $\mathrm{V}_{\mathrm{Ga}}-\mathrm{O}$ or $\mathrm{V}_{\mathrm{Ga}}-\mathrm{Al}$ should be a compensation style in the $n$-GaN bulk layer. The best fits for the Hall mobility and the Hall concentration in the bulk layer were obtained by taking the acceptor concentration $N_{A}=1.8 \times 10^{17} \mathrm{~cm}^{-3}$, the second donor concentration $N_{D 2}=1.0 \times 10^{18} \mathrm{~cm}^{-3}$, and the compensation ratio $C$ $=N_{A} / N_{D 1}=0.6$, which is consistent with Rode's theory. The saturation of carriers and low value of carrier mobility at a low temperature can also be well explained.

The relation between the MOCVD growth conditions (V/III) ratio, growth temperature, growth rate, and so on) of the crystal and the electric properties has been always a deserved research aspect. In general, high resistance or semiinsulate GaN film would be obtained if the growth circumstance is $\mathrm{N}$ rich, because the $\mathrm{N}$-rich growth condition would reduce the density of nitrogen vacancy. Nitrogen vacancy or nitrogen-site oxygen is the main source of the donor. On the other hand, the Ga-rich growth condition would decrease the intensity of Ga vacancy (a main acceptor); and increase the density of nitrogen vacancy, therefore the $n$ type would be boosted up under this condition. The relation between the growth temperature (or growth rate) and the electric properties has also been well considered by the researchers for many years. Generally, low growth rate is resulted by low growth temperature. And, too high a temperature or too low a temperature will cause problems in obtaining a film with a good crystalline structure and good electric properties. The growth process one uses should be: growing a thin buffer layer at about $500^{\circ} \mathrm{C}$ to remove the stress effect between the sapphire substrate and the $\mathrm{GaN}$ film. Then, use $1050{ }^{\circ} \mathrm{C}$ to grow the main film, because this is a proper temperature in the MOCVD method to form a high quality GaN crystalline film. However, from our measurements reported in this article, the temperature can lead to a vast atomic diffusion between the film and the substrate-this is another problem which has been neglected and needs a solution.

\section{ACKNOWLEDGMENTS}

One of the authors (X.X.) wishes to acknowledge valuable financial support from the Post Doctoral Foundation of Dr. Wong Kuon-sun under Grant No. 17 (2000) and the Science Foundation of Anhui under Grant No. 0046506. Another author (S.F.) is grateful for the financial aid from the HKU CRCG and the Hong Kong RGC research grants.

${ }^{1}$ S. Strite and H. Morkoc, J. Vac. Sci. Technol. B 10, 1237 (1992).

${ }^{2}$ S. Nakamura, T. Mukai, and M. Senoh, Appl. Phys. Lett. 64, 1687 (1994).

${ }^{3}$ S. Nakamura, M. Senoh, and S. Nagahama, Appl. Phys. Lett. 69, 4056 (1996)

${ }^{4}$ B. G. Levi, Phys. Today 49, 18 (1996).

${ }^{5}$ I. Akasaki, H. Amano, Y. Koide, K. Hiramatsu, and N. Sawaki, J. Cryst. Growth 98, 208 (1989).

${ }^{6}$ H. Amano, M. Kito, K. Hiramatsu, and I. Akasaki, Jpn. J. Appl. Phys., Part 2 28, L2112 (1989).

${ }^{7}$ S. Nakamura, T. Mukai, and M. Senoh, Jpn. J. Appl. Phys., Part 2 30, L1998 (1991).

${ }^{8}$ H. P. Maruska and J. J. Tietjin, Appl. Phys. Lett. 15, 327 (1969).

${ }^{9}$ M. Ilegems and H. C. Montgomery, J. Phys. Chem. Solids 34, 885 (1973).

${ }^{10}$ D. W. Jenkins and J. D. Dow, Phys. Rev. B 39, 3317 (1989).

${ }^{11}$ D. W. Jenkins and J. D. Dow, J. Appl. Phys. 72, 4130 (1992).

${ }^{12}$ J. Neugebauer and C. G. van de Walle, Phys. Rev. B 50, 8067 (1994)

${ }^{13}$ J. Neugebauer and C. G. van de Walle, Phys. Rev. Lett. 75, 4452 (1995).

${ }^{14}$ J. Neugebauer and C. G. van de Walle, Appl. Phys. Lett. 69, 503 (1996). 
${ }^{15}$ C. G. Van de Walle, C. Stampfl, and J. Neugebauer, J. Cryst. Growth 189, 505 (1998)

${ }^{16}$ M. A. di Forte-Poisson, F. Huet, A. Romann, M. Tordjman, D. Lancefield, E. Pereira, J. Di Persio, and B. Pecz, J. Cryst. Growth 185, 314 (1998).

${ }^{17}$ C. Wetzel, T. Suski, J. W. Auger III, E. R. Weber, and E. E. Haller, Phys. Rev. Lett. 78, 3923 (1997).

${ }^{18}$ E. Calleja, F. J. Sanchez, D. Basaka, M. A. Sanchez-Garcia, E. Munoz, I. Izpura, F. Calle, and J. L. Sanchez-Rojas, Phys. Rev. B 55, 4689 (1997).

${ }^{19}$ R. J. Molnar, T. Lei, and T. D. Moustakas, Appl. Phys. Lett. 62, 72 (1993).

${ }^{20}$ W. M. Chen, I. A. Buyanova, and M. Wagnar, Phys. Rev. B 58, R13351 (1998).

${ }^{21}$ S. Hess, R. A. Taylor, J. F. Ryan, N. J. Cain, V. Roberts, and J. Roberts, Phys. Status Solidi B 210, 465 (1998).

${ }^{22}$ C. Wezel, A. L. Chen, T. Suski, J. W. Auger III, and W. Walukiewicz, Phys. Status Solidi B 198, 243 (1996).

${ }^{23}$ S. Hess, R. A. Taylor, J. F. Ryan, B. Beumont, and P. Gibart, Appl. Phys. Lett. 73, 199 (1998)

${ }^{24}$ B. K. Meyer, D. Volm, A. Graber, H. C. Alt, T. Detchprohm, H. Amano, and I. Akasaki, Solid State Commun. 95, 597 (1995).

${ }^{25}$ U. Kauffmann, M. Kunzer, C. Merz, I. Akasaki, and H. Amano, Mater. Res. Soc. Symp. Proc. 395, 633 (1996).

${ }^{26}$ D. C. Look and D. C. Reynolds, J. Appl. Phys. 80, 2960 (1997).
${ }^{27}$ D. K. Gaskill, A. E. Wickenden, K. Doverspike, B. Tadayon, and L. R. Roland, J. Electron. Mater. 24, 1525 (1995).

${ }^{28}$ W. Gotz, N. M. Johnson, C. Chen, H. Liu, C. Kuo, and W. Imler, Appl. Phys. Lett. 68, 3144 (1996).

${ }^{29}$ D. C. Look, J. R. Sizelove, S. Keller, Y. F. Wu, U. K. Mishra, and S. P. Denbaars, Solid State Commun. 102, 297 (1997).

${ }^{30}$ D. C. Look and R. J. Molnar, Appl. Phys. Lett. 70, 3377 (1997).

${ }^{31}$ X. L. Xu, C. D. Beling, S. Fung, and Y. W. Zhao, Appl. Phys. Lett. 76, 152 (2000).

${ }^{32}$ S. Fung, X. Xiaoliang, and Z. Youwen, J. Appl. Phys. 84, 2355 (1998).

${ }^{33}$ N. F. Mott and T. D. Towse, Adv. Phys. 10, 107 (1961).

${ }^{34}$ T. Matsubara and Y. Toyozawa, Prog. Theor. Phys. 72, 651 (1996).

${ }^{35}$ X. L. Xu, Ph.D. thesis, The University of Hong Kong, 1999.

${ }^{36}$ D. C. Look, Electrical Characterization of GaAs Materials and Devices (Wiley, New York, 1989).

${ }^{37}$ B. C. Chung and M. Gershenzon, J. Appl. Phys. 72, 651 (1992).

${ }^{38}$ D. M. Hoffmann, D. Kovalev, G. Stuede, B. K. Meyer, A. Hoffmann, L. Eckey, R. Heitz, T. Detchprom, H. Amano, and I. Akasaki, Phys. Rev. B 52, 16702 (1995).

${ }^{39}$ D. K. Gaskill, L. B. Rowland, and K. Doverspike, in Properties of Group III Nitrids EMIS series No. 11, edited by James H. Edigar (1994), p. 101; and references therein. 